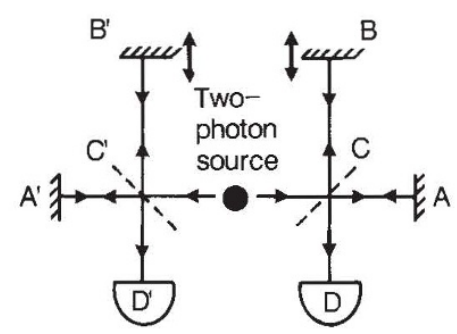

FIG. 2 Two-particle interferometer used by Kwiat et al. and Ou et al.. The arrangement resembles a pair of Michelson interferometers. The photon going left from the source can reach the detector $D^{\prime}$ via either the short route off mirror $A^{\prime}$ or the long route off moveable mirror $\mathrm{B}^{\prime}$. Similarly for the right-bound photon. The broken lines $C$ and $C^{\prime}$ indicate half-silvered mirrors. As the adjustable mirrors are moved, the rate of coincident photon arrivals varies, although the signal at the individual detectors is constant.

rate at which single photons arrive at detector $\mathrm{D}$ would depend on the relative length of paths A and B - ordinary single-particle interference would occur. But, because the source was large, the relative length of paths $A$ and $B$ was not well defined, and hence ordinary single-particle interference did not occur. Consequently, the rate of arrival at each detector in their experiment was constant independent of the mirror position. Nevertheless, the coincident arrival rate at the two detectors varied with mirror position.

Quantum mechanics accounts for this curious behaviour as follows. Because the two photons are emitted in opposite directions by the source, there are two ways for the two particles to reach the two detectors: the right-going particle takes path $\mathrm{A}$ while the left-going particle takes path $\mathrm{A}^{\prime}$; or the right-going particle takes path $\mathrm{B}$ while the left-going takes path $\mathrm{B}^{\prime}$. In this two-particle interferometer, it is interference between these two possible ways of reaching the detectors that produces the observed oscillations in the coincident arrival rate as the mirror is moved.

A different arrangement was proposed by J. Franson ${ }^{5}$ and used by P. Kwiat et al. ${ }^{2}$ and by Z. Ou et al. ${ }^{3}$ (Fig. 2). The arrangement is, again, illuminated by a downconversion source; and, again, as the mirrors are moved, the coincident arrival rate oscillates sinusoidally, even though the single-photon arrival rate at each detector is constant. For this arrangement, the photon reaching detector $\mathrm{D}$ may take the short path via mirror $\mathrm{A}$ or the long path via mirror $B$ and similarly for the other photon. But because the two photons are emitted simultaneously and detected simultaneously, it is not possible that one took the long path while the other took the short path. That is, they either both took the long path or both took the short path. The observed oscillations in the coincident arrival rate are caused by quantum mechanical interference between these two possible ways of reaching the detectors.

In what sense is the phenomenon of two-particle interference close to the heart of quantum mechanics? The answer is that quantum mechanics - but not classical physics - permits a pair of particles to be in an 'entangled state': that is, neither particle by itself is in a definite state, but there are correlations which force the pair to be in a definite, but peculiarly, quantum-mechanical state. As Schrödinger said" ${ }^{13}$, entanglement is "not one but rather the characteristic trait of quantum mechanics". For example, entanglement between apparatus and system is the essential feature behind the much discussed problem of measurement in quantum mechanics. Moreover, entanglement of spatially separated particles is the essential feature of quantum mechanics that cannot be accommodated in Einstein's 'local realist' world view.

Can the essence of entanglement be captured in everyday terms? Yes, it can. In Tapster's and Rarity's experiment, each pair of coincident photons observed at the detectors got there by travelling both paths $\mathrm{A}-\mathrm{A}^{\prime}$ and paths $\mathrm{B}-\mathrm{B}^{\prime}$. In the Franson-type experiments, each pair of observed coincident photons were emitted both early (taking the long route) and late (taking the short route). The lesson that Bell and the experimentalists have taught us about Einstein's strictly correlated but spatially separated particles is this: any attempt to follow commonsense by substituting 'either-or' for 'both-and' will necessarily fail to match the data from the two-particle interferometer.

Michael Horne is in the Department of Physics, Stonehill College, North Easton, Massachusetts 02356, USA. Abner Shimony is in the Departments of Philosophy and Physics, Boston University, Boston, Massachusetts 02215, USA. And Anton Zeilinger is in the Institut für Experimentalphysik, Universität Innsbruck, Technikerstrasse 25, A-6020 Innsbruck, Austria.

1. Rarity, J.G. \& Tapster, P.R. Phys. Rev. Lett. 64, 24952498 (1990).

2. Kwiat, P.G., Vareka, W.A., Hong, C.K., Nathel, H. \& Chiao, R.Y. Phys. Rev. A41, 2910-2913 (1990).

3. Ou, Z.Y., Zou, X.Y., Wang, L.J. \& Mandel, L. Phys. Rev. Lett. 65, 321-324 (1990)

4. Feynman, R.P. Lectures on Physics Vol. 3 (Addison Wesley, Reading, Nassachusetts, 1965).

5. Franson, J.D. Phys. Rev. Lett. 62, 2205-2208(1989).

6. Horne, M.A., Shimony, A. \& Zeilinger, A. Phys. Rev. Lett. 62, 2209-2212 (1989)

7. Ghosh, R. \& Mandel, L. Phys. Rev. Lett. 59, 1903-1905 (1987).

8. Ballentine, L.E. (ed.) Foundations of Quantum Mechanics Since the Bell Inequalities (Am. Ass. Phys. Teachers, College Park, Maryland, 1988)

9. Shih, Y.H. \& Alley, C. O. Phys. Rev. Lett. 61, 2921-2924 (1988).

10. Ou, Z.Y. \& Mandel, L. Phys. Rev. Lett. 61, 50-53 (1988).

11. Greenberger, D.M., Horne, M.A., Shimony, A. \& Zeilinger, A. Am. J. Phys. (in the press).

12. Mermin, N.D. Am. J. Phys. 58, 731-734 (1990)

13. Schrödinger, E. Proc. Camb. phit. Soc. 31, 555-563 (1935).

\section{Feather-light alloy}

ICE cubes formed in the refrigerator always have air bubbles in them. The water contains dissolved air, which is expelled on freezing. Daedalus suspects that the same sort of thing happens with metals. The upper part of a casting often has to be rejected as porous; and molten silver dissolves so much oxygen that the metal 'spits' it out as its solidifies.

So he is forcing various gases into molten metals under very high pressure. The pressure is increased still further as the metal solidifies, to keep the gas in solid solution, or at least as very tiny bubbles. The resulting supersaturated metal should be heavily stressed by its loading of occluded gas. On reheating it close to its melting point but at normal pressure, it should creep and expand, allowing the gas to emerge and foam up within it. The result: foamed metal!

DREADCO's 'Foametal'(®) will have many uses. The crucial requirement for many metal parts and components is not high strength, but high rigidity. Like foamed polystyrene, Foametal will be far lighter than fully dense material, and weight for weight far more rigid. It should be widely welcomed in aerospace engineering, car body construction and ship building (where its ability to float will be a useful bonus). The more highly foamed grades will be readily crushable by collapse of their bubbles, and should make splendid energy absorbing bumpers, helmets and so on. One very useful trick would be to sell Foametal in its gas-loaded, unannealed form. The user would merely put a slug of it in a hot mould, when it would expand, foam up and fill the mould exactly. This 'internalpressure diecasting' would be ideal for the mass production of small intricate components.

Daedalus also hopes for wide sales to the building trade, and even to do-it-yourself constructors. Foametal should feel and act much like a light but high-tenacity wood. It will be warm and friendly to the touch, and easy to saw or drill. Its crushability will let it accept nails or screws, and be bent or deformed with a hammer. Products made from it will be usefully proof against rot and fire.

Daedalus is also foaming metals with gases other than air. Mixtures containing ammonia might case-harden the inner surfaces of the bubbles by nitriding, giving a very strong Foametal indeed. Ethylene, forced into a low-melting alloy of aluminium, might polymerize under the high pressure and temperature, and fill the metal with polyethylene bubbles. Such a 'plasticized metal' would be heavily damped internally, and would absorb sound wonderfully. It could recreate our whole current crop of noisy machines in much quieter and more civilized form.

David Jones 